\title{
Structural circular birefringence and dichroism quantified by differential decomposition of spectroscopic transmission Mueller matrices from Cetonia aurata
}

\author{
Hans Arwin, A. Mendoza-Galvan, Roger Magnusson, Anette Andersson, Jan Landin, \\ Kenneth Järrendahl, E. Garcia-Caurel and R. Ossikovski
}

\section{Journal Article}

\section{Tweet}

N.B.: When citing this work, cite the original article.

Original Publication:

Hans Arwin, A. Mendoza-Galvan, Roger Magnusson, Anette Andersson, Jan Landin, Kenneth Järrendahl, E. Garcia-Caurel and R. Ossikovski, Structural circular birefringence and dichroism quantified by differential decomposition of spectroscopic transmission Mueller matrices from Cetonia aurata, Optics Letters, 2016. 41(14), pp.3293-3296.

http://dx.doi.org/10.1364/OL.41.003293

Copyright: Optical Society of America

http://www.osa.org/

Postprint available at: Linköping University Electronic Press

http://urn.kb.se/resolve?urn=urn:nbn:se:liu:diva-130379

LINKÖPINGS

UNIVERSITET 


\title{
Structural circular birefringence and dichroism quantified by differential decomposition of spectroscopic transmission Mueller matrices from Cetonia aurata
}

\author{
H. Arwin ${ }^{1, *}$, A. Mendoza-Galván ${ }^{2}$, R. Magnusson ${ }^{1}$, A. Andersson ${ }^{1}$, J. Landin ${ }^{1}$, K. \\ JÄRRENDAHL ${ }^{1}$, E. GARCIA-CAUREL ${ }^{3}$, AND R. OSSIKOVSKI ${ }^{3}$ \\ ${ }^{1}$ Department of Physics, Chemistry and Biology, Linköping University, SE-58183, Linköping, Sweden \\ ${ }^{2}$ Cinvestav-IPN, Unidad Querétaro, Libramiento Norponiente 2000, MX-76230 Querétaro, Mexico \\ ${ }^{3}$ LPICM, CNRS, Ecole Polytechnique, Université Paris - Saclay, FR-91128 Palaiseau, France \\ *Corresponding author: han@ifm.liu.se
}

Compiled June 20, 2016

Transmission Mueller-matrix spectroscopic ellipsometry is applied to the cuticle of the beetle Cetonia aurata in the spectral range $\mathbf{3 0 0 - 1 0 0 0 ~} \mathrm{nm}$. The cuticle is optically reciprocal and exhibits circular Bragg-filter features for green light. By using differential decomposition of the Mueller matrix, the circular and linear birefringence as well as dichroism of the beetle cuticle are quantified. A maximum value of structural optical activity of $\mathbf{5 6 0} \% \mathrm{~mm}$ is found. (๑) 2016 Optical Society of America

OCIS codes: (260.5430) Polarization; (260.2130) Ellipsometry and polarimetry; (160.1585) Chiral media; (160.1190) Anisotropic optical materials.

http://dx.doi.org/10.1364/ao.XX.XXXXXX

Mueller-matrix spectroscopic ellipsometry (MMSE) is established as a powerful tool to examine structural and optical properties of surfaces, thin films and multilayered materials [1, 2]. Most common are reflection studies but here we apply transmission-MMSE on the exoskeleton, here referred to as the cuticle, of the beetle Cetonia aurata. Several groups have previously applied reflection MMSE to study beetle cuticle, particularly for beetles in the Scarabaeoidea superfamily, with objective to reveal the origin of their fascinating structural colors and polarization features [3-7].

A reflection (transmission) Mueller matrix contains all reflection (transmission) properties of a sample, including its depolarization capability. It can be decomposed in various ways to get further insights into the character of the sample under investigation [8]. A sum decomposition can, as an example, provide knowledge about dominating basic reflecting mechanisms for different colors as has been shown for $C$. aurata which was shown to be a combination of a dielectric mirror and a circular polarizer $[9,10]$. For transmission Mueller matrices, a differential decomposition is feasible [11, 12]. By using this formalism it is possible to explicitly extract all birefringent and dichroic properties of a sample if it is uniform in the direction of light propagation. Here we apply differential decomposition to beetle cuticle. Such samples are structured and the obtained birefringence parameters do not correspond to intrinsic materials parameters but to effective structural parameters.

The polarizing part of a beetle cuticle has a thickness of the order of $10-50 \mu \mathrm{m}$ as observed in electron microscopy studies [13] or by analyzing optical modes in cuticle [14]. By estimating the cuticle thickness, the cumulated birefringent properties observed can be used to determine the effective birefringent properties of the structure. This will facilitate a comparison of structural optical activity and double refraction with other materials showing structural, natural or induced optical activity and/or double refraction.

The objective of this letter is to describe application of the differential matrix formalism on transmission Mueller matrices measured on beetle cuticle and to show how values of structural circular and linear birefringence/dichroic parameters can be determined. In particular we evaluate the large structural optical activity in a beetle cuticle.

The Stokes-Mueller formalism allows description and analysis of propagation of light with any degree of polarization and light-matter interaction including depolarization effects $[15,16]$. In a Cartesian $x y z$ coordinate system with $z$ as the beam propagation direction, we denote a Stokes vector $\mathbf{S}=[I, Q, U, V]^{T}$, where $T$ stands for transpose, $I$ is the total irradiance, $Q$ describes the preference for $x$ - or $y$-polarization, $U$ describes the preference for $+45^{\circ}$ - or $-45^{\circ}$-polarization and $V$ for righthanded or left-handed polarization. A Stokes vector $\mathbf{S}_{i}$ incident to an optical system is related to the emerging Stokes vector $\mathbf{S}_{o}$ by $\mathbf{S}_{o}=\tilde{\mathbf{M}} \mathbf{S}_{i}$, where $\tilde{\mathbf{M}}$ is a $4 \times 4$ matrix called the Mueller matrix. $\tilde{\mathbf{M}}$ contains all polarizing-modifying effects of a sample interacting with light, including depolarization. We here use transmission Mueller matrices normalized to the sample transmittance, i.e. the first element in the first row of $\tilde{\mathbf{M}}$. A normalized Mueller matrix $\mathbf{M}$ have elements denoted $m_{i j}(i, j=(1,4))$ 
and due to the normalization $m_{11}=1$.

A differential decomposition of $\mathbf{M}$ to find its differential matrix $\mathbf{m}$ implies determining its $\log$ arithm $\mathbf{L}=\ln \mathbf{M}$ from $d \mathbf{M} / d z=\mathbf{m} \mathbf{M}$ [11]. If $\mathbf{m}$ does not depend on $z$, i.e. $\mathbf{m}$ represents a sample which is uniform along the light propagation direction, the matrix $\mathbf{L}=\mathbf{m} d_{s}$ is the cumulated differential matrix to $\mathbf{M}$. $\mathbf{m}$ then is obtained from $\mathbf{m}=\mathbf{L} / d_{s}$ where $d_{s}$ is the sample thickness.

If the sample is non-depolarizing, $\mathbf{L}$ is Minkowskiantisymmetric and contains the isotropic absorption and all linear and circular birefringence and dichroic parameters. A generalization to a depolarizing sample results in a matrix logarithm $\mathbf{L}$ which can be decomposed as $\mathbf{L}=\mathbf{L}_{m}+\mathbf{L}_{u}$ where $\mathbf{L}_{m}$ is Minkowski antisymmetric and $\mathbf{L}_{u}$ is Minkowski symmetric $[11,12,16,17]$. $\mathbf{L}_{m}$ can be written as

$$
\mathbf{L}_{m}=\left[\begin{array}{cccc}
0 & L D & L D^{\prime} & C D \\
L D & 0 & C B & -L B^{\prime} \\
L D^{\prime} & -C B & 0 & L B \\
C D & L B^{\prime} & -L B & 0
\end{array}\right]
$$

where $L D$ and $L D^{\prime}$ are linear dichroisms along $x-y$ and $\pm 45^{\circ}$ axes, $L B$ and $L B^{\prime}$ are linear birefringences along $x-y$ and $\pm 45^{\circ}$ axes, $C D$ is circular dichroism and $C B$ is circular birefringence. Observe that the birefringence parameters are in units of radians and are cumulated values over the optical path length. Similarly the dichroic parameters are cumulated values but dimensionless. The six parameters above can also be described as three complex-valued elementary properties $P_{i}$, where $P_{1}=$ $L D+i L B, P_{2}=L D^{\prime}+i L B^{\prime}$ and $P_{3}=C D+i C B$.

The full expression for the $\mathbf{L}_{u}$ matrix within the homogeneous fluctuating medium picture is given in Refs. [16, 17].

Here we are concerned with a rotationally invariant medium in transmission and $\mathbf{L}_{u}$ reduces to

$$
\mathbf{L}_{u}=\operatorname{diag}\left[0, L D P, L D P^{\prime}, L D C\right]
$$

where $L D P=-\left\langle|\Delta L|^{2}\right\rangle-\left\langle|\Delta C|^{2}\right\rangle, L D P^{\prime}=-\left\langle|\Delta L|^{2}\right\rangle-\left\langle|\Delta C|^{2}\right\rangle$ and $L D C=-2\left\langle|\Delta L|^{2}\right\rangle$, with $\left\langle|\Delta L|^{2}\right\rangle$ being the variance for the linear properties $P_{1}$ and $P_{2}$ and $\left\langle|\Delta C|^{2}\right\rangle$ the variance for the circular properties $P_{3}$.

Samples of the scarab beetle Cetonia aurata (Linnaeus, 1758) were collected locally by one of the authors (J.L.). The left elytron (cover wing) was stripped from each specimen and a few $\mathrm{mm}^{2}$ of its inside was scraped with a small knife to remove soft tissue leaving only the endocuticle, exocuticle and epicuticle intact as seen in the cross-section in the scannning electron microscopy (SEM) image in Fig. 1. The cuticle thickness is around $50 \mu \mathrm{m}$ at the measurement position which is in the center of the elytron. However, the cuticle thickness varies between $40 \mu \mathrm{m}$ and $100 \mu \mathrm{m}$ from position to position, but the variation is mostly in the endocuticle. The exocuticle is found to be in the range $15-25 \mu \mathrm{m}$ in vicinity of the position where the MMSE measurements presented in this report were performed. By analyzing optical modes in the cuticle according to Mendoza-Gálvan et al. [14], the cuticle thickness estimated from weak interference oscillations in $m_{21}$ and $m_{24}$ were found to be $20.0 \mu \mathrm{m}$ and $20.7 \mu \mathrm{m}$, respectively.

Spectral normalized Mueller matrices were recorded with a dual rotating-compensator ellipsometer (RC2, J.A. Woollam, Co., Inc.) at normal incidence in the wavelength range $\lambda \in$ $[300-1000] \mathrm{nm}$. Focusing probes reduce the beam diameter to

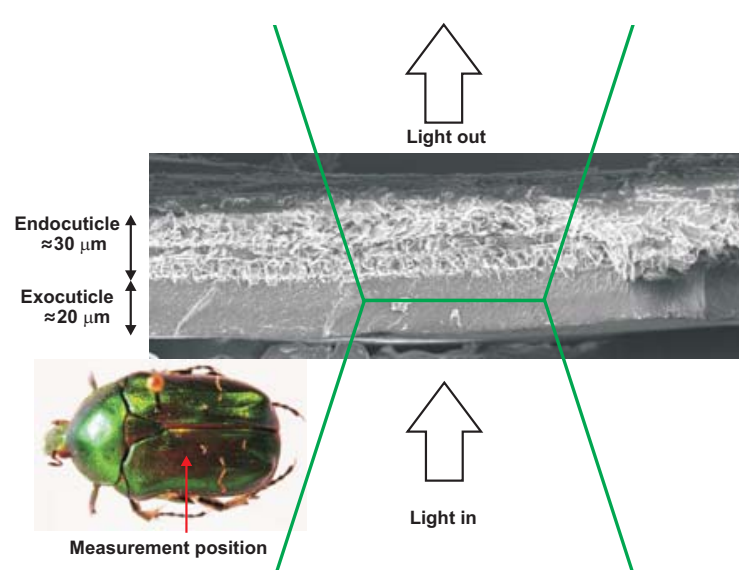

Fig. 1. SEM image of a cross section of an elytron from C. aurata with a schematic illustration of a transmission measurement through the cuticle with a focused beam (not drawn to scale). The thin $(<500 \mathrm{~nm})$ epicuticle on the exocuticle can not be seen on this scale.

$<100 \mu \mathrm{m}$. After calibration, including correction for retardation effects in the focusing probes, a straight-through measurement of a Mueller matrix in air was performed and all its elements were found to be within 0.005 of the ideal value $\mathbf{M}_{\text {air }}=\mathbf{I}$ where I is the identity matrix. Samples were mounted on a rotation stage allowing measurements at different sample azimuths.

Figure 2 shows the transmission Mueller matrix $\mathbf{M}$ of a cuticle measured from outside at an azimuth at which elements $m_{12}$ and $m_{21}$ are close to zero in average. Also shown is $\mathbf{M}^{r e v}$, i.e. the Mueller matrix measured in the reverse direction (from inside). The differences between the two matrices are that $m_{13}$, $m_{31}, m_{24}$ and $m_{42}$ change sign whereas all other elements are invariant. This symmetry implies that $\mathbf{M}$ fulfills the principle of reciprocity [18]

$$
\mathbf{M}^{r e v}=\mathbf{O M}^{T} \mathbf{O}^{-1}
$$

where $\mathbf{O}=\operatorname{diag}[1,1,-1,1]$. The difference between elements of $\mathbf{M}^{r e v}$ predicted from a measured $\mathbf{M}$ and elements of its measured reversed Mueller matrix is typically $<0.01$ but increases up to 0.05 in some elements in some spectral regions.

We can observe three spectral regions in Fig. 2: (I) below $510 \mathrm{~nm}$; (II) between 510 and $650 \mathrm{~nm}$; and (III) above $650 \mathrm{~nm}$. In region I, the cuticle can be approximated with a partial depolarizer $\mathbf{M}_{\Delta}=\operatorname{diag}[1, a, b, c]$ with $a \approx b$ and $c<a, b$. The absolute values of the off-diagonal elements in $\mathbf{M}$ are $<0.03$ except $m_{23}$ and $m_{32}$ which have values up to 0.1 . However, due to the low transmission in region I, we can not exclude that these values are systematic errors.

In region II, also referred to as the Bragg regime, the cuticle is a circular Bragg filter exhibiting circular birefringence as well as circular dichroism. In particular we notice that $m_{14} \approx m_{41}$ with values up to 0.43 . For the chosen orientation we also observe that $m_{13} \approx m_{31}$ and is non-zero with values up to 0.11 . To illustrate the polarizing properties of the cuticle we calculate some numbers for incident unpolarized light $\left(\mathbf{S}_{i}=[1,0,0,0]^{T}\right)$ with a wavelength at which $m_{41}$ is maximum. The transmitted light will have right-handed polarization with an ellipticity angle $\epsilon=1 / 2 \arcsin \left[m_{41} /\left(m_{21}^{2}+m_{31}^{2}+m_{41}^{2}\right)^{-1 / 2}\right]$ [15]. With $m_{41}=0.43, m_{31}=0.11$ and $m_{21}=-0.03$ we find an ellipticity 


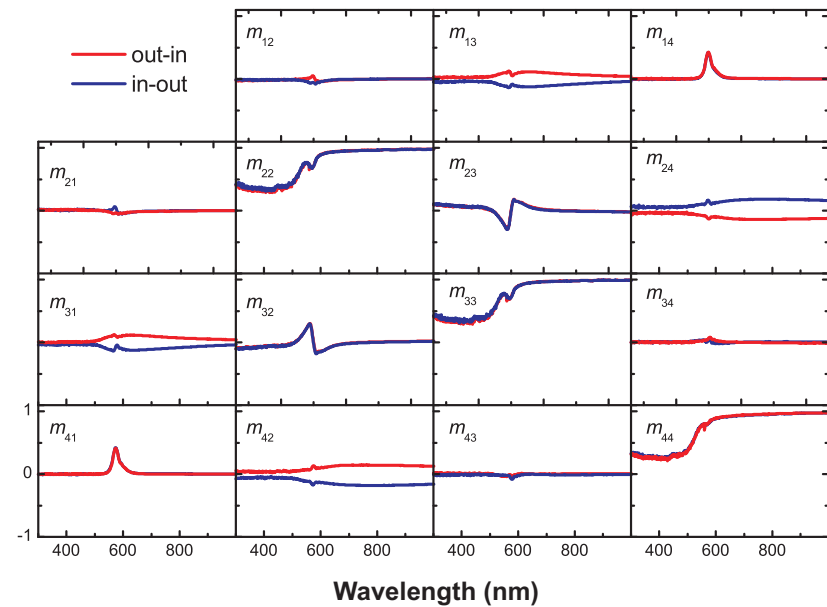

Fig. 2. Transmission Mueller matrices $\mathbf{M}$ and $\mathbf{M}^{\text {rev }}$ measured from the cuticle outside and inside, respectively, from a representative specimen.

$e=\tan \epsilon \approx 0.8$ which corresponds to near-circular polarization.

In region III, the cuticle Mueller matrix can be approximated by $\mathbf{M}=\mathbf{R}\left(-45^{\circ}\right) \mathbf{M}_{\Psi \Delta} \mathbf{R}\left(45^{\circ}\right)$ where $\mathbf{R}(\alpha)$ is the rotation matrix and $\mathbf{M}_{\Psi \Delta}$ is recognized as the standard NCS-Mueller matrix which due to the chosen sample orientation is rotated $\alpha=45^{\circ}$ [1]. The NCS-parameters in the rotated matrix are $N=\cos 2 \Psi_{t}=-m_{31}=-m_{13}, C=\sin 2 \Psi_{t} \cos \Delta_{t}=m_{22}=m_{44}$ and $S=\sin 2 \Psi_{t} \sin \Delta_{t}=-m_{24}=m_{42}$ and contain the transmission ellipsometry parameters $\Psi_{t}$ and $\Delta_{t}$ defined by $t_{p} / t_{s}=$ $\tan \Psi_{t} e^{i \Delta_{t}}$ where $t_{p}$ and $t_{s}$ are the transmission coefficients for $p$ - and s-polarization, respectively. From $N$, e.g. from element $-m_{31}$ in Fig. 2, we find that $\Psi_{t}$ is in the range $42-45^{\circ}$ and from the ratio $S / C$ we find that $\Delta_{t}$ is in the range $9-10^{\circ}$ for $\lambda \in[650,1000] \mathrm{nm}$. Element $m_{33}$ should ideally be unity but has a value slightly smaller (0.95-0.99) indicating some depolarization. In summary the cuticle for $\lambda \in[650,1000] \mathrm{nm}$ is characterized by having small retardation, diattenuation and depolarization.

To quantify birefringence and dichroic effects in the beetle cuticle, a differential decomposition of $\mathbf{M}$ in Fig. 2 is performed as described in the Theory section. The off-diagonal elements of $\mathbf{L}_{u}$ are found to be small with absolute values $<0.03$ for $\lambda<530 \mathrm{~nm}$ and $<0.005$ for $\lambda>530 \mathrm{~nm}$.

The depolarization parameters from $\mathbf{L}_{u}$ are shown in Fig. 3 and we observe that $L D P$ and $L D P^{\prime}$ are very similar. Furthermore $C D P<L D P, L D P^{\prime}$ for $\lambda<550 \mathrm{~nm}$, i.e. circular polarization is depolarized more than linear polarization. However, as discussed above, the uncertainty is large in spectral region I due to low cuticle transmittance. For $\lambda>550 \mathrm{~nm}$ all depolarization effects are much smaller and monotonically decrease with $\lambda$. The depolarization for $\lambda>550 \mathrm{~nm}$ is most probably due to scattering effects in the cuticle.

The spectral variation of the six parameters $C B, C D, L B, L D$, $L B^{\prime}$ and $L D^{\prime}$ in $\mathbf{L}_{m}$ in Eq. (1) are shown in Fig. 4. It is seen that the $x y$-linear effects $L B$ and $L D$ are small which is due to the chosen sample orientation at which the linear effects are maximum for the $\pm 45^{\circ}$-directions for which the corresponding values $L B^{\prime}$ and $L D^{\prime}$ are larger. For the circular effects we notice a band in $C D$ centered at $574 \mathrm{~nm}$. Outside this band $C D$ is zero. The corresponding Kramers-Kronig consistent feature in $C B$ also tends to zero for longer wavelengths whereas

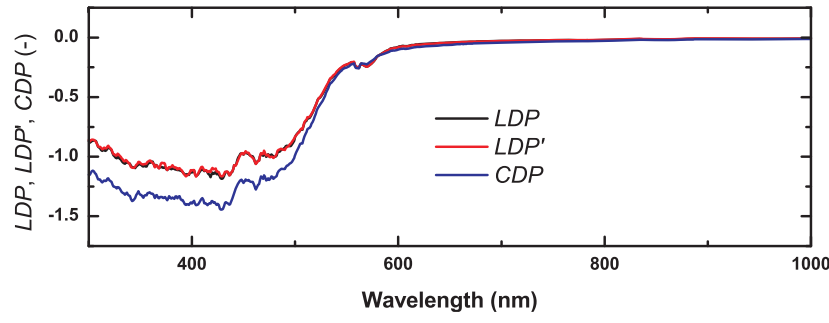

Fig. 3. Depolarizations $L D P, L D P^{\prime}$ and $C D P$ obtained from the $\mathbf{L}_{\mathcal{u}}$-matrix in Eq. (2).

for shorter wavelengths it approaches $-0.25 \mathrm{rad}$. However, for wavelengths below the Bragg regime the sample transmission is low leading to an increase of noise and also to large depolarization as discussed above. Results below $\lambda=500 \mathrm{~nm}$ should therefore be interpreted carefully as systematic errors may affect the data.
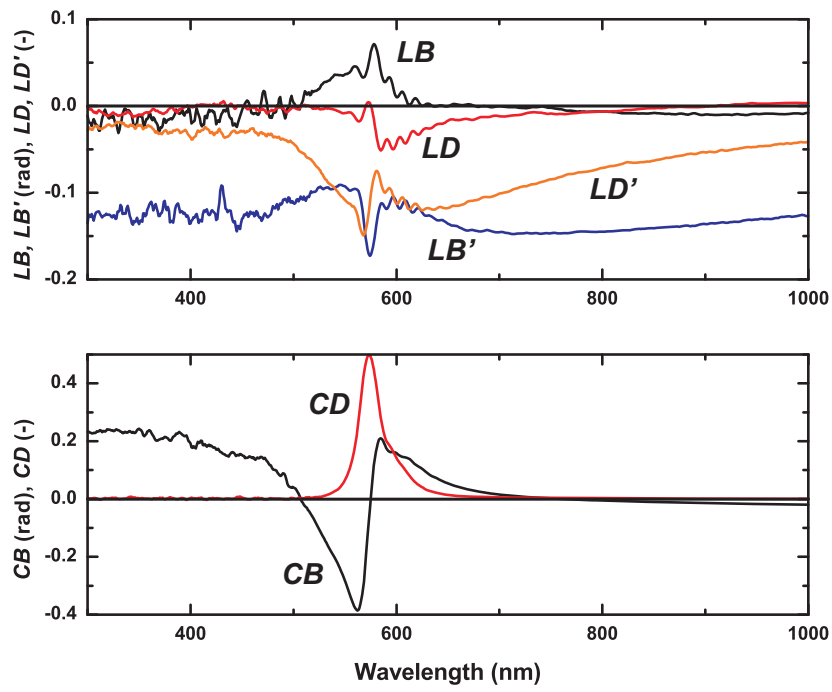

Fig. 4. $L B, L D, L B^{\prime}, L D^{\prime}, C B$ and $C D$, obtained from a differential decomposition of $\mathbf{M}$ measured in transmission through the cuticle of a C. aurata specimen.

The cuticle structure studied is an inhomogeneous medium. It has an outer thin epicuticle, an exocuticle with a helical variation in anisotropy and an inner endocuticle with assumed effective isotropic properties. The epicuticle has been found to be uniaxial with the optic axis perpendicular to the surface [19] and will thus have no influence on $\mathbf{M}$ measured in transmission at normal incidence except for a small absorption and some antireflection effects. By normalizing to the total transmission these effects are effectively eliminated. The exocuticle is the hard part of the cuticle and is composed of the polysaccharide chitin and proteins. Chitin chains form fibrils of a few $\mathrm{nm}$ in diameter. These fibrils forms in-plane lamellae which in the beetle studied here successively rotates throughout the exocuticle $[20,21]$ thus forming a helicoidal structure. The exocuticle is considered to be the part of the cuticle from which structural colors and polarizing effects originate. The endocuticle is the innermost part and is more soft and less sclerotized with a more random structure. It has a complex structure and may even contain pore canals with cytoplasmic extensions from cells inside the cuticle [20]. In SEM-images of the endocuticle of C. aurata 
$\mu \mathrm{m}$-sized fibrils are observed in layers orthogonal to each other. For the evaluation of the $\mathbf{L}_{m}$-data we assume that the endocuticle is rotationally invariant from an optical point of view.

Even though the cuticle in principle is inhomogeneous and have at least three different types of layers which each can be described with its own Mueller matrix, we assume that the measured $\mathbf{M}$ represents the exocuticle only. This is certainly correct if the epicuticle and the endocuticle are non-depolarizing and have no in-plane anisotropy. The latter is plausible but one would expect some depolarization effects, at least from the endocuticle. However, let us assume that the endocuticle is depolarizing with $\mathbf{M}_{\text {endo }}=\operatorname{diag}[1, a, a, a]$ and that $\mathbf{M}_{\text {exo }}$ represents the exocuticle. Simple algebra shows that $\mathbf{M}=\mathbf{M}_{\text {endo }} \mathbf{M}_{\text {exo }}$ will become asymmetric with $m_{14}=a m_{41}$. Similarly if $\mathbf{M}=$ $\mathbf{M}_{\text {exo }} \mathbf{M}_{\text {endo }}$ is analyzed we find $m_{41}=a m_{14}$. These two cases correspond to sending light through the cuticle in reverse directions. However, experimentally we observe that it holds for $\mathbf{M}$ that $m_{41}=m_{14}$ for all rotations and for both directions of light through the cuticle. We conclude that $\mathbf{M}$ is a good representation for the exocuticle only.

If $\mathbf{M}$ stem from the exocuticle only, we can evaluate $\mathbf{L}_{m}$ further. The exocuticle is inhomogeneous as it is a periodic layered helicoidal structure. The observed circular birefringence and dichroism originate from internal linear birefringence in consecutively rotated lamellae. Thus there is no molecular optical activity but we may, from the observed structural circular birefringence, deduce an effective optical activity, i.e. circular birefringence per unit length for the cuticle material to facilitate comparison with optically active materials [16]. From Fig. 4 we find that $|C B|$ at $\lambda=562 \mathrm{~nm}$ has an extreme value of around $0.39 \mathrm{rad}\left(22^{\circ}\right)$ and with an exocuticle thickness $d_{s}=20 \mu \mathrm{m}$, we find a specific rotation of $1 / 2|C B| / d_{s} \approx 560 \% \mathrm{~mm}$. This should be compared with rotations observed in Optical Rotatory Dispersion (ORD) studies which are of the order of $0.01^{\circ}$, with natural optical activity in quartz causing a specific rotation of around $25 \% \mathrm{~mm}$ at $\lambda=550 \mathrm{~nm}$ [22] and with liquid crystals which can rotate the polarization several thousands of $\% \mathrm{~mm}$ [23].

Finally we compare $L B^{\prime}$ to the retardation $\Delta_{t}$ determined from $\mathbf{M}_{\Psi \Delta}$ in region III for the $\pm 45^{\circ}$ principal axes. From Fig. 4 we find that $L B^{\prime}$ is in the range $0.14-0.15$ rad which corresponds to $8-9^{\circ}$ in excellent agreement with $\Delta_{t}$ considering that depolarization was ignored for $\Delta_{t}$.

In summary, a differential decomposition of Mueller matrices measured in transmission mode on the cuticle of C. aurata reveals circular structural birefringence and dichroism centered around the circular Bragg resonance in the twisted layered structure of the cuticle. Linear birefringence and dichroism were observed in the Bragg resonance spectral region as well as for wavelengths up to $1000 \mathrm{~nm}$. Linear birefringence is also observed in the spectral range $(300-550 \mathrm{~nm})$ but due to low cuticle transmission the uncertainty is large in this spectral range. Cuticle depolarization was evaluated from the variances of the elementary polarizing properties and found to be small above the Bragg resonance but decreases for wavelengths shorter than the Bragg resonance but also here the low transmission makes the uncertainty large. A maximum value of the specific rotation of the cuticle was found to be $560 \% \mathrm{~mm}$.

Acknowledgements James Stuhr is acknowledged for guidance to perform transmission Mueller-matrix measurements. Lia Fernàndez del Río is acknowledged for performing SEM.

Financial support is acknowledged from the Swedish Government Strategic Research Area in Materials Science on Func- tional Materials at Linköping University, Carl Tryggers Foundation, Knut and Alice Wallenberg foundation, the Mexican Council for Science and Technology (Conacyt) and the Minister of Public Education (SEP).

\section{REFERENCES}

1. H. Fujiwara, Spectroscopic Ellipsometry: Principles and Applications (John Wiley \& Sons, Ltd, 2003).

2. E. Garcia-Caurel, R. Ossikovski, M. Foldyna, A. Pierangelo, B. DrevilIon, and A. De Martino, "Advanced Mueller ellipsometry instrumentation and data analysis," in "Ellipsometry at the Nanoscale,", M. Losurdo and K. Hingerl, eds. (Springer Berlin Heidelberg, 2013), chap. 2, pp. 31143.

3. D. Goldstein, Appl. Opt. 45, 7944 (2006).

4. I. Hodgkinson, S. Lowrey, L. Bourke, A. Parker, and M. W. McCall, Appl. Opt. 49, 4558 (2010).

5. H. Arwin, R. Magnusson, J. Landin, and K. Järrendahl, Phil. Mag. 92 , 1583 (2012).

6. L. Fernàndez del Río, H. Arwin, and K. Järrendahl, Thin Solid Films 571, 410 (2014).

7. O. Arteaga, Thin Solid Films, in press (2016).

8. R. Ossikovski, M. Anastasiadou, S. Ben Hatit, E. Garcia-Caurel, and A. De Martino, Phys. Stat. Sol. (a) 205, 720 (2008).

9. H. Arwin, R. Magnusson, E. Garcia-Caurel, C. Fallet, K. Järrendahl, M. Foldyna, A. De Martino, and R. Ossikovski, Opt. Express 23, 1951 (2015).

10. R. Ossikovski, M. Foldyna, C. Fallet, and A. De Martino, Opt. Lett. 34, 2426 (2009).

11. R. Ossikovski, Opt. Lett. 36, 2330 (2011).

12. O. Arteaga and B. Kahr, Opt. Lett. 38, 1134 (2013).

13. A. C. Neville, Biology of the Arthropod Cuticle (Springer-Verlag, 1975).

14. A. Mendoza-Gálvan, E. Muñoz-Pineda, H. Arwin, and K. Järrendahl, Opt. Mat. Express 4, 2484 (2014).

15. E. Colett, Polarized Light: Fundamentals and Applications (Marcel Dekker, 1993)

16. J. J. Gil and R. Ossikovski, Polarized Light and the Mueller Matrix Approach (CRC Press, 2016).

17. R. Ossikovski and O. Arteaga, Opt. Lett. 39, 4470 (2014).

18. A. Schonhöfer and H.-G. Kuball, Chem. Phys. 115, 159 (1987).

19. H. Arwin, T. Berlind, B. Johs, and K. Järrendahl, Opt. Express 21, 22646 (2013).

20. R. F. Chapman, The Insects: Structure and Function (Cambridge University Press, 1998)

21. T. Lenau and M. Barfoed, Adv. Eng. Mat. 10, 299 (2008).

22. R. C. Weast, Handbook of Chemistry and Physics (CRC Press, 1972).

23. V. A. Belyakov, V. E. Dmitrienko, and V. P. Orlov, Sov. Phys. Usp. 22, 63 (1979). 


\section{FULL REFERENCES}

1. H. Fujiwara, Spectroscopic Ellipsometry: Principles and Applications (John Wiley \& Sons, Ltd, 2003).

2. E. Garcia-Caurel, R. Ossikovski, M. Foldyna, A. Pierangelo, B. Drevillon, and A. De Martino, "Advanced Mueller ellipsometry instrumentation and data analysis," in "Ellipsometry at the Nanoscale," , M. Losurdo and K. Hingerl, eds. (Springer Berlin Heidelberg, 2013), chap. 2, pp. 31-143.

3. D. Goldstein, "Polarization properties of scarabaeidae,", Appl. Opt. 45, 7944-7950 (2006).

4. I. Hodgkinson, S. Lowrey, L. Bourke, A. Parker, and M. W. McCall, "Mueller-matrix characterization of beetle cuticle:polarized and unpolarized reflections from representative architectures," , Appl. Opt. 49, 4558-4567 (2010).

5. H. Arwin, R. Magnusson, J. Landin, and K. Järrendahl, "Chiralityinduced polarization effects in the cuticle of scarab beetles: 100 years after michelson,", Phil. Mag. 92, 1583-1599 (2012).

6. L. Fernàndez del Río, H. Arwin, and K. Järrendahl, "Polarizing properties and structural characteristics of the cuticle of the scarab beetle Chrysina gloriosa," , Thin Solid Films 571, 410-415 (2014).

7. O. Arteaga, "Natural optical activity vs circular bragg reflection studied by Mueller matrix ellipsometry,", Thin Solid Films. -, 0-0 (2016).

8. R. Ossikovski, M. Anastasiadou, S. Ben Hatit, E. Garcia-Caurel, and A. De Martino, "Depolarizing mueller matrices: how to decompose them?" , Phys. Stat. Sol. (a) 205, 720-727 (2008).

9. H. Arwin, R. Magnusson, E. Garcia-Caurel, C. Fallet, K. Järrendahl, M. Foldyna, A. De Martino, and R. Ossikovski, "Sum decomposition of mueller-matrix images and spectra of beetle cuticles,", Opt. Expr. 23, 1951-1966 (2015).

10. R. Ossikovski, M. Foldyna, C. Fallet, and A. De Martino, "Experimental evidence for naturally occurring nondiagonal depolarizers," , Opt. Lett. 34, 2426-2428 (2009).

11. R. Ossikovski, "Differential matrix formalism for depolarizing anisotropic media," , Opt. Lett. 36, 2330-2332 (2011).

12. O. Arteaga and B. Kahr, "Characterization of homogenous depolarizing mediabased on mueller matrix differential decomposition," , Opt. Lett. 38, 1134-1136 (2013).

13. A. C. Neville, Biology of the Arthropod Cuticle (Springer-Verlag, 1975).

14. A. Mendoza-Gálvan, E. Muñoz-Pineda, H. Arwin, and K. Järrendahl, "Evidence for a dispersion relation of optical modes in the cuticle of the scarab beetle Cotinis mutabilis," , Opt. Mat. Exp. 4, 2484-2496 (2014).

15. E. Colett, Polarized Light: Fundamentals and Applications (Marcel Dekker, 1993).

16. J. J. Gil and R. Ossikovski, Polarized Light and the Mueller Matrix Approach (CRC Press, 2016).

17. R. Ossikovski and O. Arteaga, "Statistical meaning of the differential mueller matrix of depolarizing homogeneous media," , Opt. Lett. 39, 4470-4473 (2014).

18. A. Schonhöfer and H.-G. Kuball, "Symmetry properties of the mueller matrix,", Chem. Phys. 115, 159-167 (1987).

19. H. Arwin, T. Berlind, B. Johs, and K. Järrendahl, "Cuticle structure of the scarab beetle Cetonia aurata analyzed by regression analysis of mueller-matrix ellipsometricdata," , Opt. Expr. 21, 22646-22656 (2013).

20. R. F. Chapman, The Insects: Structure and Function (Cambridge University Press, 1998).

21. T. Lenau and M. Barfoed, "Colours and metallic sheen in beetle shells, a biomimetic search for material structuring principles causing light interference," , Adv. Eng. Mat. 10, 299-314 (2008).

22. R. C. Weast, Handbook of Chemistry and Physics (CRC Press, 1972).

23. V. A. Belyakov, V. E. Dmitrienko, and V. P. Orlov, "Optics of cholesteric liquid crystals," , Sov. Phys. Usp. 22, 63-88 (1979). 